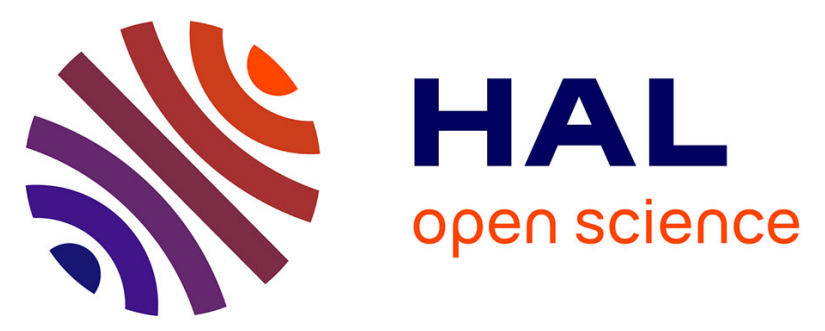

\title{
Distributed Identical Grating Sensing System Oriented to Equipment Intelligent Sense in Cloud Manufacturing
}

Quan Liu, Kunchao Bao, Yilin Fang, Tao Huang, Zhengying Li

\section{To cite this version:}

Quan Liu, Kunchao Bao, Yilin Fang, Tao Huang, Zhengying Li. Distributed Identical Grating Sensing System Oriented to Equipment Intelligent Sense in Cloud Manufacturing. IFIP International Conference on Advances in Production Management Systems (APMS), Sep 2015, Tokyo, Japan. pp.371-378, 10.1007/978-3-319-22759-7_43. hal-01431118

\section{HAL Id: hal-01431118 \\ https://hal.inria.fr/hal-01431118}

Submitted on 10 Jan 2017

HAL is a multi-disciplinary open access archive for the deposit and dissemination of scientific research documents, whether they are published or not. The documents may come from teaching and research institutions in France or abroad, or from public or private research centers.
L'archive ouverte pluridisciplinaire HAL, est destinée au dépôt et à la diffusion de documents scientifiques de niveau recherche, publiés ou non, émanant des établissements d'enseignement et de recherche français ou étrangers, des laboratoires publics ou privés. 


\title{
Distributed Identical Grating Sensing System Ori- ented to Equipment Intelligent Sense in Cloud Manufacturing
}

\author{
Liu Quan, Bao Kunchao*, Fang Yilin, Huang Tao, Li Zhengying** \\ Key Laboratory of Fiber Optic Sensing Technology and Information Processing, Ministry of \\ Education, Wuhan University of Technology, Wuhan, Hubei 430070, China
}

Abstract. The intelligent sense of equipment is a key link in cloud manufacturing. Identical fiber Bragg gratings (FBG) have a flexible multiplexing number and are unaffected by the laser's bandwidth. A distributed identical grating system oriented to cloud manufacturing equipment's intelligent sense is developed by combining wavelength tunable laser and broadband laser source in this paper. The system collects the reflect spectroscopy to calculate the center wavelength of each FBG by Gaussian fitting. Some optimization is also done to the Gaussian fitting algorithm to increase the demodulation speed in this paper. In the system, 100 reflective FBGs of only $0.1 \%$ reflection with the interval space of $5 \mathrm{~m}$ are connected to a long fiber. All the central wavelengths and positions of the FBGs can be measured. The results show that the linearity of the center wavelength with temperature is up to $99.8 \%$ in range of $-10^{\circ} \mathrm{C}$ to $60^{\circ} \mathrm{C}$.

Keywords: fiber optic sensor; intelligent sense; cloud manufacturing.

\section{Introduction}

Cloud Manufacturing is a new-emerging and prospective manufacturing mode which has remarkable advantages such as providing flexible service, high cooperativity and knowledge integration level ${ }^{[1]}$. The physical resource layer, which is the fundamental layer in the structure of cloud manufacturing, consists of a large number of manufacturing equipment ${ }^{[2]}$. Because of the diversity, large number of equipment and adverse working environment, the monitoring data is massive and diverse ${ }^{[3]}$. Fiber Bragg Grating (FBG) with its compactness, electromagnetic immunity and excellent multiplexing capability ${ }^{[4-5]}$ has become a research focus in optical sensing fields. FBGs are

\footnotetext{
* First corresponding author:baokunchao@whut.edu.cn

** Second corresponding author:zhyli@whut.edu.cn

The research is supported by the Specialized Research Fund for the Doctoral Program of Higher Education of China (Grant No. 20120143110017), and the Wuhan International Scientific and Technological Cooperation Project (Grant No. 2014030709020306).
} 
easy to form a sensor network for distributed measurement and are widely used in structural health monitoring system for huge buildings such as bridges, tunnels, dams ${ }^{[6]}$. Therefore, a distributed FBG sensing system with large capacity and high sensitivity is an effective solution to raise the performance of cloud manufacturing equipment intelligent sense.

According to different methods of multiplexing, there are mainly three kinds of distributed FBG sensing system: wavelength division multiplexing (WDM) mode ${ }^{[7]}$, time division multiplexing (TDM) mode and frequency division multiplexing (FDM) mode. Because of the bandwidth limit of laser source, generally no more than 20 FBGs can be multiplexed on a single fiber in WDM mode ${ }^{[8]}$. FDM mode cannot achieve a long distance monitoring due to the coherence length of laser ${ }^{[9]}$. Using high reflectivity FBG, TDM mode is unable to realize a large multiplexing number of FBGs as a result of the crosstalk and shadow effect between FBGs. In the 10th references article, there are 4 FBGs of $6 \%$ reflection multiplexed on a single fiber ${ }^{[10]}$. In the 11th references article, there are 9 FBGs of $3 \%$ reflection multiplexed on a fiber

${ }^{[11]}$. In the 12th references article, there are 1000 identical FBGs of $0.001 \%$ reflection multiplexed on a fiber ${ }^{[12]}$. And in the 13th references article, 20 identical FBGs of $0.01 \%$ reflection are multiplexed on one fiber ${ }^{[13]}$. A lower reflection means a lower insertion loss and negligible crosstalk and a larger multiplexing number. Therefore, we use the low reflection FBG to increase the FBG multiplexing number. In this paper, we developed a distributed identical FBG system oriented to cloud manufacturing equipment's intelligent sense by combining the wavelength tunable laser and the broadband laser source. In experiments, 100 reflective FBGs of only $0.1 \%$ reflection with the interval space of $5 \mathrm{~m}$ are connected to a long fiber. In order to insure the FBG wavelength's demodulation accuracy, we adopt the Gaussian fitting algorithm to calculate the center wavelength of each FBG according to the reflect spectrum. Gaussian fitting algorithm is complex and very time -consuming, we also do some optimization to the algorithm to improve demodulation rate.

\section{Research Content and Experiment Results}

\subsection{System Design and Implementation}

In theory, the FBG reflective spectrum is similar to Gaussian distribution. If FBG reflective spectrum is known, the center of the spectrum (also the center wavelength of FBG) can be calculated by Gaussian fitting.

The pulse modulated light with a stepping and periodic wavelength goes through the identical FBG serial array on a single fiber. Different wavelength the input light has, FBG reflects light with different power. When the input light's wavelength is closer to the center wavelength of FBG, the optical pulse reflected by FBG is stronger. On the contrary, father the wavelength between input light and the center wavelength of FBG is, the reflected light is weaker.Therefore, acquiring all the reflected pulses for each FBG over a period, we can piece together the spectral envelope of each FBG. Then, Gaussian fitting method is used to calculate the center wavelength of each FBG according to the envelope. 


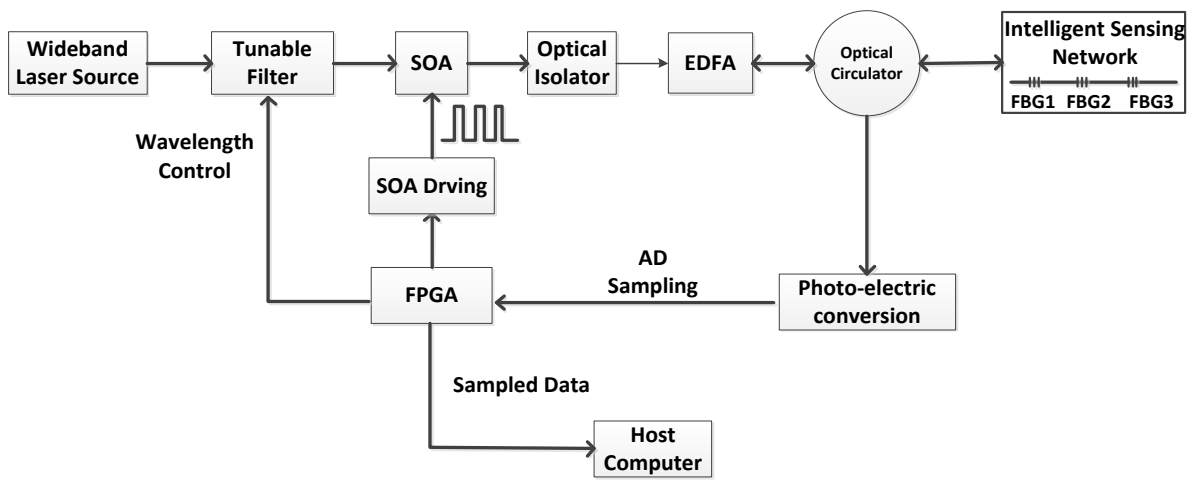

Fig. 1. Schematic configuration of the sensing system

Fig.1 shows the specific architecture of the sensing system we design. The output of wideband laser source is light with various wavelengths. These wavelengths are screened out by a tunable filter controlled by a field programmable gate array (FPGA) processer. The FPGA processer also controls the semiconductor optical amplifier (SOA) driving circuit to produce a periodic pulse sequence to drive the SOA. The light out from the SOA module is pulsed and periodic in wavelength. In order to increase the multiplexing FBG's number, we need to increase the power of the light input. So after the isolator, we adopt an Erbium-doped optical fiber amplifier (EDFA) to amplify the light intensity. Then the light goes through the FBG array on one fiber, the optical pulses reflected by FBGs go into the photo-electric conversion module via the optical circulator. The photon-electric conversion module transforms the optical signal to electrical signal, and the electrical signal are transformed to digital signal and acquired by the FPGA processor. At last, the sampling data is uploaded to a host computer which runs a Gaussian-fitting-method based program to handle the data and then calculates the center wavelength of each FBG. As for the location of each FBG, with optical time domain reflection technology, we can easily figure out the FBG's location by time difference between optical pulses.

\subsection{FBG Wavelength Demodulation}

In experiments, 100 reflective FBGs of only $0.1 \%$ reflection with the interval space of $5 \mathrm{~m}$ are connected to a long fiber. In theory, all the 100 FBGs have a same wavelength about $1552.5 \mathrm{~nm}$ at room temperature. At the time of manufacture, the long fiber with 100 identical FBGs is wrapped around a spool manually. Because of the nonuniformity of stress, 100 FBGs' center wavelengths are not the same. Fig.2 shows their differences. Therefore, the input light's wavelength is in the range of $1552.000 \mathrm{~nm}$ to $1553.2 \mathrm{~nm}$, the pulse width is about $10 \mathrm{~ns}$, and the wavelength step is $10 \mathrm{pm}$. After restructuring all reflected spectrums, we can get the spectral envelope of each FBG like Fig.3 shows. 


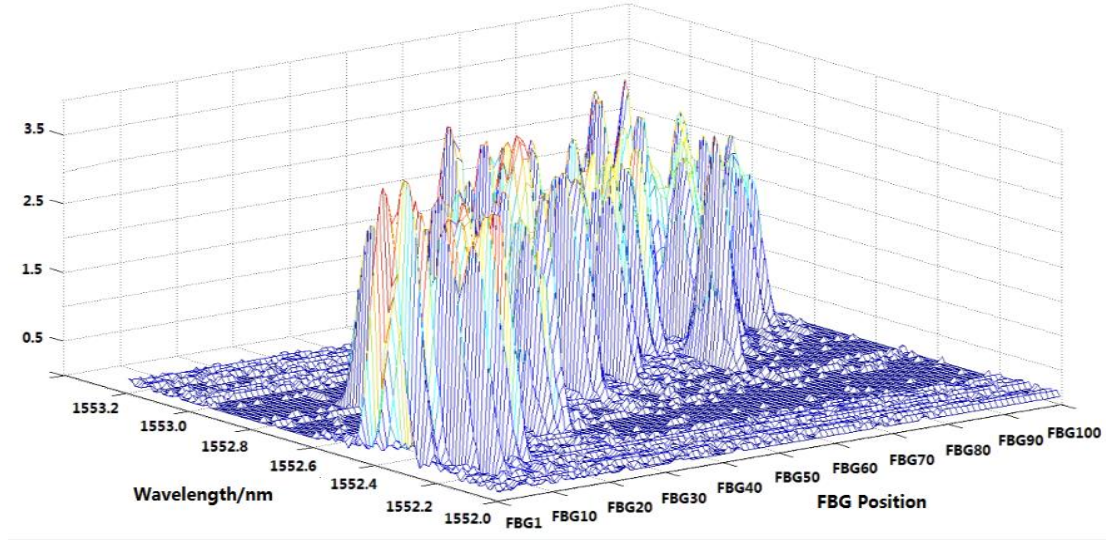

Fig. 2. 100 FBGs' spectrum distribution

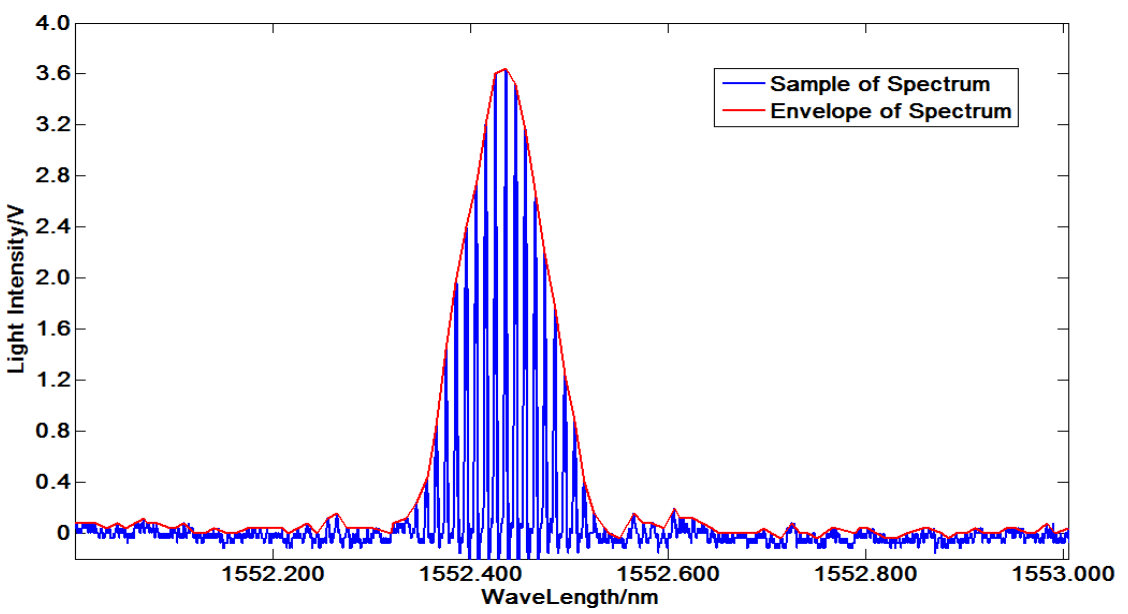

Fig. 3. Envelope of a FBG's reflect spectrum

The refection spectrum of FBG can be approximately described as the following formula:

$$
\mathrm{I}(\lambda)=\mathrm{I}_{0} \exp \left[-4 \ln 2\left(\frac{\lambda-\lambda_{\mathrm{s}}}{\Delta \lambda_{\mathrm{s}}}\right)\right]
$$

In formula (1), $I_{0}$ represents the power peak of the reflect spectrum; $\lambda_{s}$ represents the wavelength when the spectrum power is $\mathrm{I}_{0} . \Delta \lambda_{\mathrm{s}}$ is the $3 \mathrm{~dB}$ bandwidth of reflect spectrum. As Fig.4 shows, the black dots are the reflect spectrum points, and the red line is a Gaussian fitting curve line according to the black points. The spread center of the Gaussian curve now represents the center wavelength of FBG. 


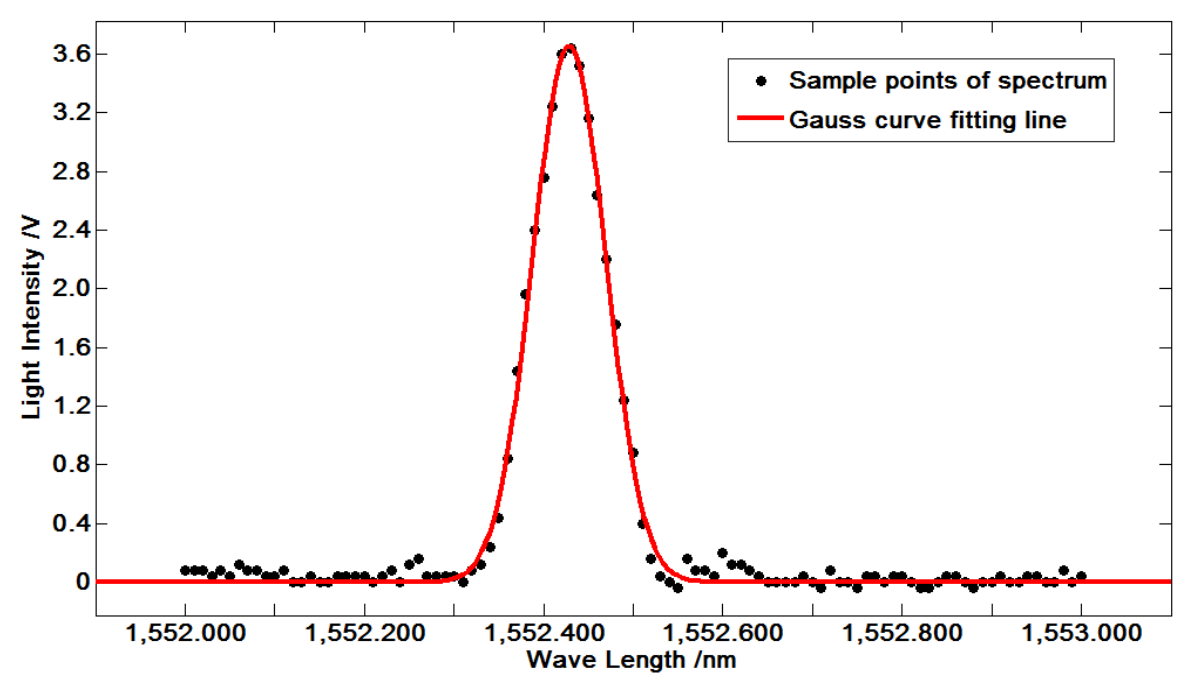

Fig. 4. Gaussian fitting of spectrum

In the process of Gaussian fitting, we convert the non-linear Gaussian fitting methods to polynomial fitting methods which is linear and simpler. Therefore, the least square theory could be used to deduce the calculating formula for wavelength demodulation. Firstly, some transformations should be done to the formula (1) in order to change the formula into a linear form, through which the fitting efficiency will be improved. Let:

$$
\begin{gathered}
\mathrm{y}=\ln I \\
\mathrm{a}=-4 \ln 2 /\left(\Delta \lambda_{s}\right)^{2} \\
\mathrm{a}=-4 \ln 2 /\left(\Delta \lambda_{s}\right)^{2} \\
\mathrm{c}=\ln I_{0}-4 \ln 2 * \lambda_{s} /\left(\Delta \lambda_{s}\right)^{2}
\end{gathered}
$$

Then the equation in formula (1) can be converted to the following:

$$
\mathrm{y}=\mathrm{a} \lambda^{2}+b \lambda+c
$$

So now the least square principle will help to calculate the values of modulus a, b, c. According to the equation (3), the sum of squared residuals is given by:

$$
\mathrm{S}=\sum_{i=1}^{n}\left(y_{i}-a \lambda_{i}^{2}-b \lambda_{i}-c\right)^{2}
$$

According to the least square principle, in order to achieve the minimum value of $\mathrm{S}$, we take derivatives of $\mathrm{S}$ with respect to $\mathrm{a}, \mathrm{b}$ and $\mathrm{c}$ and let the derivatives equal to zero as the following equation shows.

$$
\begin{aligned}
& \sum_{i=1}^{n} y_{i}=a\left(\sum_{i=1}^{n} \lambda_{i}{ }^{2}\right)+b\left(\sum_{i=1}^{n} \lambda_{i}\right)+n c \\
& \sum_{i=1}^{n} y_{i}=a\left(\sum_{i=1}^{n} \lambda_{i}{ }^{2}\right)+b\left(\sum_{i=1}^{n} \lambda_{i}\right)+n c
\end{aligned}
$$




$$
\sum_{i=1}^{n} \lambda_{i}{ }^{2} y_{i}=a\left(\sum_{i=1}^{n} \lambda_{i}^{4}\right)+b\left(\sum_{i=1}^{n} \lambda_{i}^{3}\right)+c\left(\sum_{i=1}^{n} \lambda_{i}^{2}\right)
$$

Working out the equation (5), the value of $a, b, c$ will be known. Then a and $b$ are fed into formula (6) to obtain the center wavelength value of FBG reflect spectrum that is $\lambda_{0}$ given by:

$$
\lambda_{0}=-b /(2 a)
$$

On the other side, one time wavelength demodulation of the whole sensing system with 100 FBGs costs at least 100 times Gaussian fitting process. The whole process contains many similar or same calculation procedures. We can simplify the Gaussian fitting process by working out the results of these similar calculation procedures thus making our fitting algorithm more efficient in time and improving the demodulation rate obviously. According to the derivations above, every fitting needs one logarithmic process done and a three-variable linear equation (5) be worked out. Given the number of sample points we need, there exists 100 time logarithmic processes, 708 times float number's addition and subtraction operations and 517 times float number multiplications. For all FBGs, the frequency sweeping range of input light are the same, in this paper the range is $1552.000 \mathrm{~nm} \sim 1553.2 \mathrm{~nm}$, and the step wavelength also the same (10pm),that means the value of $\lambda_{\mathrm{i}}$ in equation (5) for each FBG is the same. Therefore, for each FBG's fitting process, the results of $\left(\sum_{\mathrm{i}=1}^{\mathrm{n}} \lambda_{\mathrm{i}}^{4}\right) 、\left(\sum_{\mathrm{i}=1}^{\mathrm{n}} \lambda_{\mathrm{i}}^{3}\right) 、\left(\sum_{\mathrm{i}=1}^{\mathrm{n}} \lambda_{\mathrm{i}}^{2}\right) 、\left(\sum_{\mathrm{i}=1}^{\mathrm{n}} \lambda_{\mathrm{i}}\right)$ are the same. These results above can be worked out in advance and stored in the computer's ram so that they can directly be used in the subsequent operations. After these calculation optimization, given the same sample points number 100, one time fitting process needs 100100 time logarithmic processes and only 308 times float number's additions and subtractions and 310 times float number's multiplications, thus reducing 400 times float number's additions and subtractions and 310 times float number's multiplications.

In the experiments, we use a normal personal computer with a CPU frequency of $2.27 \mathrm{GHz}, 4 \mathrm{~GB}$ memory size and a 32-bit Windows 7 operating system. A Gaussian fitting program is developed within VS2010 developing tools to execute the wavelength demodulation process. Before the optimization above, 10000 times demodulations cost nearly 0.2 second. And after the optimization, 1000 times demodulations cost only 0.025 second. The experimental results show that, the optimization methods put forward in this paper has improved the fitting efficiency obviously and raise the demodulation rate of TDM FBG distributed sensing network up to more than $10 \mathrm{~Hz}$.

\subsection{Temperature Monitoring Experiments}

The single long fiber where 100 only $0.1 \%$ reflection FBGs grating on with an interval of 5 meters is wrapped around a spool. Then the spool is put into an incubator chamber. The temperature of the incubator chamber changes from $-10{ }^{\circ} \mathrm{C}$ to $60{ }^{\circ} \mathrm{C}$ with a step of $5{ }^{\circ} \mathrm{C}$. At each temperature point, we acquire a set of sampling data to demodulate each FBG's wavelength. Fig 5 shows the relationship between demodulated wavelengths of 1th FBG, 25th FBG, 50th FBG, 75th FBG, 100th FBG and the 
linear changing temperature. A good linear relationship can be seen from Fig 5 between wavelength and temperature and the linearity degree is up to $99.8 \%$.

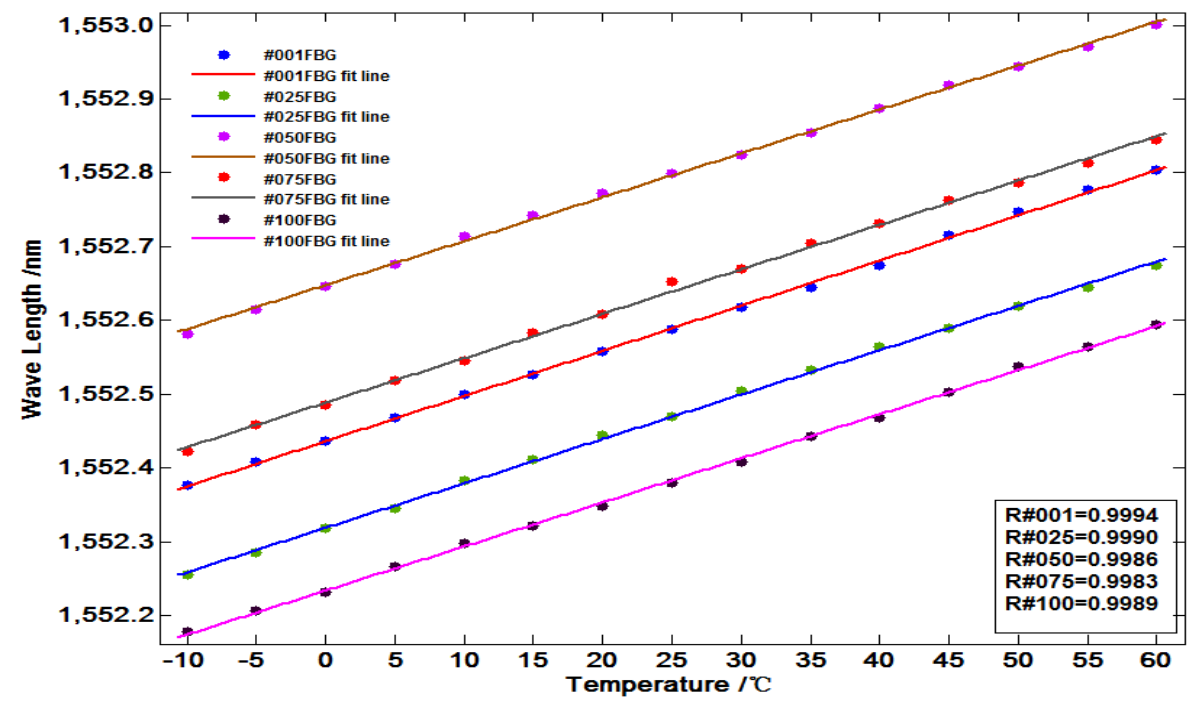

Fig. 5. relationship between wavelength and temperature

\section{Conclusion}

In this paper, a distributed equipment intelligent sense system in cloud manufacturing taking use of identical FBGs is developed and implemented. In the system, 100 reflective FBGs of only $0.1 \%$ reflection with the interval space of $5 \mathrm{~m}$ are connected to a long fiber. All the central wavelength and position of the FBGs can be measured simultaneously. The results shows that the linearity of the center wavelength with temperature is up to $99.8 \%$ in range of $-10^{\circ} \mathrm{C}$ to $60^{\circ} \mathrm{C}$. On the other side, this paper also provides an effective optimization method to improve the demodulation rate of the system. The distributed identical grating system designed in this paper will play an active role in the equipment intelligent sense field in cloud manufacturing because of its characteristics such as high capacity and strong interference immunity.

\section{References}

1. Lei Ren, Lin Zhang, Fei Tao, Chun Zhao, Xudong Chai, Xinpei Zhao. Cloud manufacturing: from concept to practice[J]. Enterprise Information Systems, 2015, Vol.9 (2), pp.186-209.

2. ZHOU Zude, LIU Quan, XU Wenjun. From digital manufacturing to cloud manufacturing $[\mathrm{J}]$. International Journal of Engineering innovation and Management. 
3. LI Rui-fang, LIU Quan, Xu Wen-jun. Perception and access and adaptation of equipment resources in cloud manufacturing[J]. Computer Integrated Manufacturing Systems.2012. Vol.18 NO.7.

4. Hui XU, Wei-Xin Ren, Zuo-Cai Wang. Deflection Estimation of Bending Beam Structures Using Fiber Bragg Grating Strain Sensors[J]. Advances in Structurak Engineering, 2015, Vol.18(3).

5. KAREN W, TIMOTHY B, ROBERT R, et al. Fiber optic sensors for health monitoring of morphing airframes : I. Bragg grating strain and temperature sensor [J]. Smart Materials Structures, 2000, 9 (2):163-169.

6. Xuefeng Zhao, Jie Lu, Ruicong Han, et. Application of Multiscale Fiber Optical Sensing Network Based on Brillouin and Fiber Bragg Grating Sensing Techniques on Concrete Structures

7. JIANG Desheng, FAN Dian, Mei Jiachun. Multiplexing/Demultiplexing Technology Based on Fiber Bragg Grating Sensors [J]. Laser\&Optoelectronics Progress. 2005. Vol42,NO.4.

8. Song Jianfei, Shao Liyang, Zhang A-ping, et al. New FBG based dynamic-strain interrogation system[J]. Journal of Optoelectronics Laser, 2007,18(8):924-926.

9. Li Zhaoyun, Li Su, Liu Zhiqiang,et al.. Research in optical frequency domain reflectometry fiber Bragg grating distributed strain sensor[J]. Optical Communication Technology,2012,36(12):33-25.

10. Zhang Manliang, Sun Qizhen, Wang Zi, Li Xiaolei, Liu Hairong, Liu Deming. Investigation on Distributed Optical Fiber Sensor Based on Identical-low-reflective Fiber Gratings [J]. Laser\&Optoelectronics Progress. 2011. Vol 08.

11. Zhang Yanjun, Xie Xiaopeng, Bi Weihong. High-Speed High-Multiplexing Distributed Temperature Sensor Network Based on Weak-Reflection Fiber Gratings [J]. Chinese Journal of Lasers. 2013. Vol. 40, No. 4.

12. Y Wang, J Gong, B Dong, et al. . A large serial time-division multiplexed fiber Bragg grating sensor network[J]. J Lightwave Technol,2012,30(17):2751-2756.

13. Zhang Caixia, Zhang Zhenwei, Zheng Wanfu, Liu Xiaohang, etc. Study of a QuasiDistributed Optical Fiber Sensing System Based on Ultra-Weak Fiber Bragg Gratings [J]. Chinese Journal of Lasers. 2014. Vol. 41, No. 4. 